\title{
Fatores associados à formação da aliança terapêutica na psicoterapia obrigatória
}

\author{
Natacha Hennemann de Oliveira Becker \\ Clínica Cruz e Hennemann Médicos e Associados \\ Sílvia Pereira da Cruz Benetti \\ Universidade do Vale do Rio dos Sinos
}

\begin{abstract}
Resumo
O objetivo deste estudo foi identificar de que forma se estabelece a aliança terapêutica (AT) em pacientes encaminhados de forma obrigatória para a psicoterapia, em comparação aos pacientes que buscam atendimento psicoterapêutico voluntariamente, verificando-se também as características das representações de objeto, mecanismos de defesa, percepção de coerção, e gravidade dos sintomas. Participaram da pesquisa 51 sujeitos, sendo 15 casos em psicoterapia obrigatória e 36 casos em tratamento voluntário. Ambos os grupos foram selecionados em uma instituição pública de saúde que oferece psicoterapia para funcionários públicos municipais. Os resultados apontaram que apesar dos pacientes obrigatórios apresentarem sintomas mais graves, foi possível o estabelecimento da AT. Além disso, o status de paciente obrigatório não influenciou o estabelecimento da AT e sim a percepção de coerção na procura de atendimento.
\end{abstract}

Palavras-chave: aliança terapêutica; psicoterapia; psicologia.

\begin{abstract}
Factors associated with the formation of the therapeutic alliance in mandatory psychotherapy. The aim of this study was to identify how the therapeutic alliance (TA) is established in patients referred for mandatory psychotherapy compared to patients who seek psychotherapy voluntarily. It was also verified o the characteristics of object representations, defense mechanisms; perception of coercion and severity of symptoms. Participants were 51 subjects, divided into a psychotherapy mandatory group $(N=15)$, which was compared with a group of patients in voluntary treatment $(N=36)$. Both groups were selected from a public health institution that provides psychotherapy for municipal employees, both voluntary and mandatory. The results showed that although mandatory patients showed more symptoms, it was possible to establish the TA. Moreover, the mandatory status did not influence the establishment of TA, but the perception of coercion when seeking treatment.
\end{abstract}

Keywords: therapeutic alliance; psychotherapy; psychology.

\section{Resumen}

Factores asociados con la formación de la alianza terapéutica en la psicoterapia obligatoria.

El objetivo de este estudio fue identificar la forma de establecer la alianza terapéutica (AT) en pacientes referenciados para la psicoterapia obligatoria en comparación con los pacientes que voluntariamente buscan atención psicoterapéutica, además de verificar las características de representaciones de objetos, mecanismos de defensa, la coerción percibida, y la gravedad de los síntomas. Participaron en el estudio 51 sujetos, 15 casos de psicoterapia obligatoria y 36 en el tratamiento voluntario. Ambos grupos fueron reclutados de una institución de salud pública que proporciona la psicoterapia para los empleados municipales. Los resultados mostraron que a pesar de los pacientes obligatorios tenían síntomas más graves, fue posible establecer la AT. Además, la situación de paciente obligatorio no influyó en la creación de la AT y sí, la percepción de coerción en la búsqueda de atención.

Palabras clave: alianza terapéutica; psicoterapia; psicología.

A crescente demanda de atendimentos psicoterápicos não voluntários ou compulsórios tem exigido dos profissionais da psicologia uma adequação das intervenções terapêuticas dirigidas para esses casos. Atualmente, ocorrem diversas situações em que a psicoterapia não é uma escolha do paciente, mas uma obrigação. Nesses casos, há uma exigência ISSN (versão eletrônica): 1678-4669 de atendimento obrigatório/involuntário originada de diversas demandas judiciais, tais como: situações envolvendo crianças vítimas de abuso sexual, famílias em situação de vulnerabilidade social, cuidadores que estão na eminência de perder o poder familiar de seus filhos, homens que agrediram fisicamente suas companheiras, dependentes químicos graves, doentes mentais Acervo disponivel em: www.scielo.br/epsic 
graves, dentre outros. Outra demanda são as situações em que os pacientes são encaminhados por escolas, instituições públicas, instituições empregadoras, familiares e/ou cônjuges. Nesses casos, ainda que sem demanda judicial, a busca pelo atendimento também não é espontânea e existe uma exigência para que o paciente permaneça em atendimento.

A relação não voluntária foi definida por Thibaut e Kelley em 1959 (citado em Chui \& Ho, 2006) como sendo composta pelos seguintes elementos: quando o paciente se sente forçado a permanecer nela, seja por coação psicológica ou legal, inexistindo alternativas válidas ou atrativas; quando o paciente não pode deixar o tratamento porque o custo de deixá-lo é muito alto; e quando a pessoa acredita estar em desvantagem nessa relação por não ter melhores alternativas de escolha.

Por sua vez, em um processo psicoterapêutico existem condições consideradas mais propícias para a obtenção de resultados satisfatórios, como a motivação do paciente. Segundo Greenson (1981) e Yoshida (2001), a motivação seria o que levaria o paciente a uma psicoterapia, visando superar sua doença e sensação de desamparo. Além disso, a motivação constituiria a origem da aliança terapêutica, considerada o vínculo positivo e consciente entre paciente e terapeuta (Bordin, 1979), por esse motivo é que o paciente estaria racionalmente disposto a colaborar e seguir as recomendações do terapeuta.

Além da motivação, existem outros fatores de indicação para uma psicoterapia que podem ou não contribuir na obtenção de resultados satisfatórios. Dessa forma, segundo Yoshida (2001), tanto a escolha do modelo teórico quanto condições específicas para o tratamento, bem como os recursos internos do paciente, do terapeuta e a qualidade da relação estabelecida entre eles (aliança terapêutica), influenciam no processo psicoterápico. Portanto, o vínculo estabelecido entre paciente e terapeuta pode modificar a motivação, que pode ser mantida, aumentada ou diminuída. Cabe então ao terapeuta proporcionar as condições necessárias para acolher empaticamente o material trazido pelo paciente, para que juntos possam desenvolver uma boa aliança terapêutica (Yoshida, 2001).

Todavia, como se estabeleceria a aliança terapêutica nas situações obrigatórias? Considerando a aliança terapêutica como um fator fundamental para o estabelecimento da relação terapêutica, além de um conceito comum e panteórico em psicoterapia, neste trabalho buscamos compreender alguns fatores que estariam associados ao estabelecimento desse vínculo quando o paciente é obrigado a iniciar uma psicoterapia e permanecer em atendimento psicoterapêutico, independente de sua vontade. Cabe destacar que na literatura, são identificados os termos psicoterapia compulsória, psicoterapia obrigatória e involuntária. No primeiro caso trata-se de uma determinação judicial, no segundo, de uma determinação interna de uma instituição ou de decisão de outra pessoa que não o paciente. Neste trabalho, utilizaremos os termos compulsório e obrigatório, como sinônimos de um atendimento involuntário, visto que em ambos a necessidade/ demanda de atendimento foi identificada por um 'outro', que exige tratamento como condição para que o suposto paciente possa obter algum benefício.

\section{Psicoterapia obrigatória}

Diversos países têm adotado modalidades de tratamento psicoterapêutico não voluntário, seja como uma medida para tratar patologias mentais graves (através de tratamento ambulatorial e hospitalização), seja para tentar tratar pessoas envolvidas com o sistema de justiça criminal, tais como: abusadores sexuais infantis, usuários de álcool e drogas, dentre outros (Opjordsmoen et al., 2010; Viadel, Nicolás, Prieto, Galatayud, \& Beneyto, 2010).

O tratamento psicoterapêutico ambulatorial involuntário é uma prática frequente para com pacientes portadores de doença mental grave em vários países como EUA, Canadá, Reino Unido, Nova Zelândia, França, Alemanha, Áustria e Israel. Na Espanha, esse tipo de prática é utilizada desde 2003, a partir da criação de uma legislação específica. O tratamento ambulatorial involuntário - TAI ou ordens de tratamento compulsórias - CTOs, consistem em diferentes tipos de medidas judiciais sem internação que tem por objetivo fazer com que pacientes com doença mental grave permaneçam em atendimento, evitando as re-hospitalizações e abandono do tratamento (Nakhost, Perry, \& Frank, 2012; Viadel et al., 2010).

Uma pesquisa realizada pelo serviço de psiquiatria do Hospital Clínico Universitário de Valência avaliou a eficácia do tratamento ambulatorial involuntário (Viadel et. al., 2010). Foi investigado se pacientes psiquiátricos com tratamento ambulatorial involuntário (TAI) reduziam o uso de serviços de saúde mental, em comparação com um grupo controle não submetido a essa medida judicial. O resultado indicou que os pacientes com TAI tiveram uma redução no número de vezes que precisaram utilizar os serviços de urgência e no número de internações e tempo de permanência de cada internação. Em contrapartida, essa redução não foi significativamente maior, se comparada ao grupo controle, quando foram realizados os ajustes quanto ao diagnóstico, sexo, idade e uso prévio dos serviços hospitalares. Quando analisada, após o ajuste dos dados, ao ser avaliada através da redução do uso de serviços hospitalares, a efetividade do TAI ficou comprometida e estatisticamente não se mostrou mais eficaz. Mesmo em vista de tal resultado, o TAI foi extremamente eficaz quando os critérios de avaliação foram a aderência ao tratamento durante o período da medida, a satisfação do paciente e a evolução desse paciente após medida judicial.

Outra pesquisa que sugere a efetividade do tratamento ambulatorial involuntário foi realizada por Nakhost et al. (2012). Nesse trabalho foram avaliados pacientes psiquiátricos que tinham sido submetidos à Ordens de Tratamento Comunitárias (CTOs) durante um período de nove anos em dois hospitais universitários canadenses (McGill University Hospitals). Os resultados apontaram uma significativa redução no número de re-hospitalizações durante o período da medida de tratamento (CTOs).

No Brasil, uma pesquisa realizada com homens violentos, que foram obrigados a frequentar o atendimento psicoterápico para não serem presos (por terem agredido fisicamente suas companheiras), Reis e Ramos (2010) verificaram que os participantes iniciam o atendimento contra a sua vontade, mostrando-se desconfiados e confusos quanto ao trabalho a ser realizado. Entretanto, no decorrer das sessões, percebeu-se que a demanda existia, mas 
era sufocada, porque os participantes ainda não confiavam nos terapeutas. Após estabelecerem uma relação de confiança com os terapeutas, estes puderam investir no trabalho. Ao final dos atendimentos realizados, percebeu-se que, mesmo a partir de uma demanda compulsória, promoveu-se um trabalho que resultou em reflexões e investimento desses homens em suas vidas e relacionamentos.

No atendimento psicoterapêutico obrigatório, portanto, existem inúmeros fatores que podem contribuir ou dificultar o andamento e resultado do tratamento. Entre esses fatores está a percepção de coerção (Kaltiala-Heino, Laippala, \& Salokangas, 1997). Isto é, o quanto o paciente, mesmo tendo sido referido de forma compulsória ao atendimento, sente-se realmente coagido a procurar a psicoterapia. Dessa forma, o grau do impacto da coerção no resultado do tratamento está associado com pior resultado de tratamento e evolução do paciente. Podem se manifestar sentimentos negativos no paciente quanto maior o caso de coerção, além de expectativas negativas sobre o resultado do tratamento, dificultando o estabelecimento de uma relação de confiança entre o paciente e os profissionais no tratamento (Kaltiala-Heino et al., 1997). Nessa direção, Iversen, Hoyer e Sexton (2007) avaliaram a percepção legal e objetiva de coerção dos pacientes com doenças psiquiátricas agudas, internados em um hospital psiquiátrico universitário norueguês. O estudo apontou que $51 \%$ dos pacientes admitidos involuntariamente relataram baixos níveis de percepção de coerção, enquanto que 34\% relataram altos níveis de coerção, sendo que $18 \%$ desses foram expostos a situações objetivas de coerção (uso da força física).

Nesse contexto de atendimento psicoterapêutico obrigatório/compulsório, observa-se que a literatura é inconclusiva, apontando estudos com resultados contraditórios quanto à efetividade de medidas de atendimento como TAI e CTOs (Frank, Perry, Kean, Sigman, \& Geagea, 2005). Dessa forma, algumas das situações supracitadas expõem casos de atendimentos psicoterapêuticos que obtiveram êxito, mesmo sendo compulsórios. Esses exemplos demonstram que a obrigatoriedade de tratamento nem sempre é sinônimo de fracasso. No entanto, sabe-se que na prática clínica, a obrigatoriedade do tratamento pode dificultar o estabelecimento do vínculo entre paciente e terapeuta, tornando a intervenção mais complexa, devido a fatores como a falta de solicitação de ajuda, a inexistência de um motivo e/ou a negação do problema (Fritz, 2006). Nesse caso, está em questão o estabelecimento da aliança terapêutica (AT), que é entendida como a relação/vínculo que se estabelece entre paciente e terapeuta durante o tratamento.

\section{A AT na psicoterapia obrigatória}

Conforme Bordin (1979), a AT é composta por três características essenciais: 1) acordo nos objetivos do tratamento entre paciente e terapeuta, 2) acordo nas tarefas e 3) desenvolvimento do vínculo (confiança e apego) entre a dupla. Por sua vez, o estabelecimento da aliança nas situações de psicoterapia obrigatória é influenciado por diversos fatores que fortalecem ou não o estabelecimento do vínculo entre o terapeuta e o paciente (Sotero \& Relvas, 2012).
A obrigatoriedade da participação do sujeito no atendimento, segundo Ribeiro et al. (2010), não impede o engajamento e investimento necessário para que os objetivos sejam alcançados. Mas é tarefa do terapeuta motivar e envolver o paciente, a ponto de transformar a obrigação em demanda. Além disso, existe uma série de variáveis do paciente que também influenciam na AT. Três fatores do paciente têm sido identificados como impactantes na qualidade da aliança: a severidade do problema, o tipo de prejuízo e a qualidade das relações de objeto ou apego (Horvarth \& Luborsky, 1993). Outros autores também destacam as características de personalidade do paciente. Nesse sentido, a personalidade é apontada como determinante na qualidade da AT, principalmente o padrão defensivo estabelecido pelo paciente. Entretanto algumas pesquisas (Despland et al., 2001; Gomes, Ceitlin, Hauck, \& Terra, 2008; Kramer, Roten, Perry, \& Despland, 2009) identificaram que o padrão defensivo isoladamente não influenciou na qualidade da AT inicial. Além disso, apesar de alguns pacientes utilizarem mecanismos de defesa muito primitivos, foi possível o estabelecimento de uma aliança terapêutica de qualidade.

Aspectos intrapsíquicos como a qualidade das representações de objeto (QRO) também podem ser considerados um importante indicador de melhorias da sintomatologia e disfunção que levaram o paciente a buscar tratamento psicoterapêutico. Estudos identificam que as representações de objeto podem ser um significativo preditor de AT, e de resultados positivos da terapia (Byrd, Patterson, \& Turchik, 2010; Goldman \& Anderson, 2007; Hersoug et al., 2011; Piper, Ogrodniczuk, \& Joyce, 2004). Pacientes com melhor qualidade nas representações de objeto têm uma capacidade maior de tolerar ansiedades e frustrações despertadas durante o processo terapêutico. Entretanto, algumas pesquisas não identificaram relação entre a qualidade das relações de objeto como influente no estabelecimento da $\mathrm{AT}(\mathrm{Pi}$ per et al., 2004; Vans et al., 2008).

Com base nessas questões, este estudo tem por objetivo identificar e comparar o estabelecimento da aliança terapêutica em pacientes encaminhados à psicoterapia de forma obrigatória, em relação aos pacientes que buscam atendimento psicoterapêutico voluntariamente. Além disso, os objetivos específicos foram de comparar as representações de objeto e mecanismos de defesa, verificar a percepção de coerção e analisar a gravidade dos sintomas dos pacientes em relação às características da AT nos dois grupos investigados.

\section{Método}

O método utilizado foi de uma pesquisa quantitativa através de um delineamento de levantamento transversal, descritivo e comparativo.

\section{Amostra}

Os participantes foram 51 sujeitos de um grupo naturalmente formado de servidores municipais, que fizeram psicoterapia no Instituto de Previdência e Assistência de um município da região da grande Porto Alegre, entre abril de 2012 e março de 
2013. A escolha da amostra se deu por conveniência, de acordo com a procura e encaminhamento para o serviço de psicoterapia dessa instituição. A modalidade de atendimento oferecida na instituição é psicoterapia breve psicanalítica. Foram avaliados 36 pacientes que buscaram a psicoterapia voluntariamente e 15 pacientes encaminhados de forma obrigatória. Os participantes foram: servidores públicos municipais afastados do trabalho por um diagnóstico psiquiátrico, estes encaminhados pelo médico psiquiatra para psicoterapia obrigatória e servidores municipais que procuraram espontaneamente o serviço para iniciarem um processo psicoterapêutico. No momento do atendimento, todos os participantes haviam sido medicados pelo médico psiquiatra e não estavam em situação de internação hospitalar. Os diagnósticos em ambos os grupos incluíam casos de depressão e bipolaridade.

\section{Instrumentos}

Escala de aliança psicoterápica da Califórnia - CALPAS P (versão do paciente). A escala CALPAS (Marziali \& Alexander, 1991) foi validada no Brasil por Marcolino e Iacoponi (2001). O instrumento inclui uma versão do paciente $(\mathrm{P})$, do terapeuta $(\mathrm{T})$ ou por avaliadores treinados (R). Os itens da Calpas-P recebem uma pontuação que varia de um (absolutamente não) a sete (totalmente), sendo que itens que apresentam frases com sentido negativo tivessem seus índices refletidos. No presente trabalho utilizou-se a versão do paciente, englobando 24 itens que avaliam quatro dimensões: compromisso do paciente (PC) que reflete o aspecto relacional da AT, capacidade de trabalho do paciente (PWC) que evidencia o componente de colaboração ativa inerente a AT, compreensão e envolvimento do terapeuta (TUI) que revela a contribuição deste para a AT, acordo entre o paciente e o terapeuta (WSC) quanto aos objetivos e às estratégias do tratamento. A pontuação total da escala foi obtida pelo cálculo da média da pontuação das quatro subescalas da CALPAS. A consistência interna (Alpha de Cronbach) para a Calpas-P foi de 0,90 para a escala total.

Bell object relations and reality testing inventory (BORRTI - Forma O). Foi traduzido e adaptado por Bruscato e Iacoponi (2000) e abrange todo o espectro de funcionamento de relações objetais. Constituiu-se num inventário auto administrável, com 45 declarações descritivas que o sujeito marca como verdadeiro ou falso, de acordo com sua experiência mais recente. O inventário se compõe de quatro subescalas para os quatro fatores: dimensões alienação (AL), vinculação insegura (IN), egocentrismo (EGO), incapacidade social ( $\mathrm{SOC}$ ). A correção do teste é realizada através de um programa de computador específico. A consistência interna (Alpha de Cronbach) foi de 0,59 para a escala total.

Defense style questionnaire - DSQ-40 (Andrews, Singh, \& Bond, 1993). Para verificar o padrão defensivo, foi utilizada a forma traduzida e adaptada para o português validada por Blaya et al., (2004). Este instrumento é composto por 40 perguntas relacionadas à descrição das defesas contidas no DSM-III-R, subdivididas em três fatores: maduro, neurótico e imaturo. Cada pergunta é avaliada em uma escala de um a nove, em que um indica "discordo totalmente" e nove indica "concordo totalmente". O DSQ-40 pode fornecer escores para as 20 defesas individuais e para os três fatores (maduro, neurótico e imaturo). Os escores das defesas individuais são calculados pela média dos dois itens de cada mecanismo de defesa dado e os fatores dos escores são calculados pelos escores médios das defesas que pertencem a cada fator. A consistência interna (Alpha de Cronbach) foi de 0,90.

Escala de sintomas (SCL-90-R) (Derogatis, 1994). A SCL90-R é uma medida de autorrelato que avalia padrões de sintomas escalas globais (Índice de Gravidade Geral, Índice de Perturbação Sintomática Positiva, e Total de Sintomas Positivos) e nove escalas de sintomas (somatização, obsessão-compulsão, sensibilidade interpessoal, depressão, ansiedade, hostilidade, ansiedade fóbica, ideação paranóide e psicoticismo). Para o cálculo dos escores, somam-se os pontos de 0 a 4 de cada escala aos sete itens adicionais e divide-se pelo número de itens de cada dimensão. Para calcular o IGG (Índice de Gravidade Geral), é preciso somar os pontos das nove dimensões sintomáticas aos sete itens adicionais e dividi-los pelo número de itens respondidos na escala. Os índices de consistência interna (Alpha de Cronbach) variam entre 0,80 (somatização) e 0,89 (depressão).

Juntamente com os instrumentos, foi entregue a cada participante um questionário com apenas duas perguntas, baseadas no MacArthur Admission Experience Scale - AES-15 (Iversen, Hoyer, Sexton, \& Gronli, 2002), sem adaptação para o português, instrumento que avalia a percepção de coerção do paciente sobre seu tratamento. As perguntas feitas aos pacientes incluíram questões sobre a percepção de coerção do encaminhamento e a necessidade de atendimento.

\section{Procedimentos de coleta de dados}

Foi marcado antes do início da psicoterapia um horário distinto ao da primeira sessão para que os pacientes respondessem aos seguintes instrumentos: o BORRTI - Forma O, o DSQ-40 e a Escala de Sintomas - SCL-90-R, os quais foram aplicados pela Assistente Social do serviço. Ao final da quinta sessão, os pacientes responderam ao CALPAS-P e ao questionário que avalia percepção de coerção ao tratamento baseado no MacArthur Admission Experience Scale-AES-15. Os instrumentos foram entregues pela assistente social do serviço, portanto, em horário distinto ao da terapia. Dois terapeutas realizaram os atendimentos, tanto obrigatórios como não obrigatórios.

\section{Considerações éticas}

O projeto de pesquisa foi encaminhado ao Comitê de Ética em Pesquisa da Universidade do Vale do Rio dos Sinos (UNISINOS) e teve sua aprovação no dia 16 de março de 2012, sob o número do Projeto 12/006. Também foi encaminhado ao Conselho Municipal de Previdência e Assistência do município para autorização de execução, tendo sua aprovação no dia 22 de fevereiro de 2012.

\section{Procedimentos de análise dos dados}

Os dados coletados foram analisados pelo programa estatístico SPSS $₫$ versão 19. Foram realizadas análises descritivas, considerando as variáveis categóricas e contínuas. Em seguida, foram verificadas as diferenças dos grupos em psicoterapia obrigatória x psicoterapia não obrigatória, bem como em relação à percepção de coerção para realizar psicoterapia, pacientes que independente 
da forma do encaminhamento, obrigatório ou não, declararam sentir-se obrigados a realizar psicoterapia (percepção positiva de coerção) x pacientes que não se sentem obrigados (percepção negativa de coerção). Os testes Qui-Quadrado e o teste $t$ de Student foram empregados e o nível de significância adotado foi de $p$ menor ou igual a 0,05 .

\section{Resultados}

Do total de 59 pacientes em acompanhamento psicológico no período de coleta, três não concordaram em participar do estudo (um caso em psicoterapia obrigatória e dois casos em psicoterapia não obrigatória) e cinco abandonaram a psicoterapia antes do quinto encontro (pacientes em psicoterapia não obrigatória). A amostra final foi constituída por 51 pacientes em psicoterapia, com idade média de 42,2 anos $(D P=11,02)$. No que tange a escolaridade, $37,3 \%$ tinham o ensino médio completo, seguido por $35,3 \%$ que declararam possuir ensino superior completo.

No grupo de psicoterapia obrigatória (GO), as idades variaram entre 28 a 51 anos $(M=37,57, D P=6,86)$. Neste grupo, predominaram as participantes do sexo feminino (86,7\%). Já no grupo de pacientes em psicoterapia não obrigatória (GNO), a idade média foi de 44 anos $(D P=11,86)$, variando de 25 a 70 anos. Todos os participantes deste grupo eram do sexo feminino.

\section{Análise comparativa entre os grupos obrigatório e não obrigatório - aliança terapêutica}

De acordo com os resultados comparativos da Escala de Aliança Psicoterápica da Califórnia (CALPAS-P) em relação ao grupo obrigatório (GO) e não obrigatório (GNO), verifica-se que os grupos não diferiram significativamente em relação ao escore total do instrumento e, também em suas subescalas (ver Tabela 1). Dessa forma, esses resultados indicam que o desenvolvimento da AT foi similar nos dois grupos.

\section{Escala de sintomas (SCL-90-R)}

Os resultados comparativos da Escala de Sintomas (SCL90-R) indicam que O Índice de Gravidade Geral (IGG) dos sintomas dos pacientes do grupo de psicoterapia obrigatória (GO) $(M=1,77, D P=0,79)$ é significativamente superior à média do grupo em psicoterapia não obrigatória (GNO) $(M=1,31, D P=$ $0,69, p=0,045)$. Nas escalas de sintomas, as médias do grupo em psicoterapia obrigatória (GO) são também superiores: obssessividade $(M=2,14, D P=0,73)$, depressão $(M=2,10, \mathrm{DP}=0,85, p=$ $0,050)$, hostilidade $(M=2,93, D P=0,73, p=0,013)$ e ansiedade fóbica $(M=1,47, D P=1,00, p=0,018)$, quando comparadas aos pacientes em situação não obrigatória (GNO): obssessividade ( $M$ $=1,69, D P=0,73)$, depressão $(M=1,60, \mathrm{DP}=0,79)$, hostilidade $(M=2,43, D P=0,59)(p=0,013)$ e ansiedade fóbica $(M=0,81$, $D P=0,82)$.

\section{Mecanismos defensivos (DSQ-40)}

Em relação ao questionário de estilos de defesa DSQ-40 não existem diferenças significativas nas médias dos fatores maduro (FM), neurótico (FN) e imaturo (FI), entre os grupos em psicoterapia obrigatória (GO) (fator maduro (FM) $\mathrm{M}=4,42 \mathrm{DP}$ = 1,27, fator neurótico (FN) $M=4,35 D P=1,28$, e fator imaturo (FI) $M=3,96, D P=1,39)$ e em psicoterapia não obrigatória (GNO) (fator maduro (FM) $M=4,94, D P=1,48$, fator neurótico (FN) $M=4,47 D P=1,68$ e fator imaturo (FI) $M=3,43, D P=1$, 13).

\section{Relações objetais}

Por último, a avaliação das características das relações objetais na escala alienação (AL), vinculação insegura (IN), egocentrismo (EGO) e incapacidade social (SOC), realizada através do inventário BORRTI indicou que, dentre os 15 casos do grupo obrigatório (GO), a frequência de escore clínico é: nove (60\%) casos clínicos na escala alienação, seis casos clínicos (40\%) na escala vinculação insegurança, nove (60\%) casos clínicos na escala egocentrismo e três (20\%) casos clínicos na escala de incapacidade social. Quanto aos 38 casos não obrigatórios (GNO), verificase: 21 (63\%) casos clínicos na escala alienação, 17 casos clínicos (41\%) na escala vinculação insegurança, 13 (40\%) casos clínicos na escala egocentrismo e 12 (36\%) casos clínicos na escala de incapacidade social. Houve diferença significativa somente na escala egocentrismo, no grupo obrigatório (GO) apresentando um número maior de casos $(9,60 \%)$ com escore clínico nesta escala $\left(X^{2}(1)=9,487, p=0,004\right)$.

\section{Tabela 1}

Resultados Comparativos da Escala de Aliança Psicoterápica da Califórnia

\begin{tabular}{cccccc}
\hline & Grupo & N & Média & DP & Significância \\
\hline $\begin{array}{c}\text { Escala de Aliança Psi- } \\
\text { coterápica da Califórnia } \\
\text { - Total }\end{array}$ & Obrigatório & 15 & 21,18 & 4,93 & \\
Não obrigatório & 36 & 23,15 & 3,12 & 0,183 \\
$\begin{array}{c}\text { Compromisso do Paciente } \\
\text { (PC) }\end{array}$ & Obrigatório & 15 & 5,17 & 1,44 & \\
& Não obrigatório & 36 & 5,69 & 1,02 & 0,164 \\
$\begin{array}{c}\text { Capacidade de trabalho } \\
\text { (PWC) }\end{array}$ & Obrigatório & 15 & 4,80 & 1,24 & \\
Não obrigatório & 36 & 5,03 & 0,91 & 0,550 \\
$\begin{array}{c}\text { Compreensão e envolvi- } \\
\text { mento do terapeuta (TUI) }\end{array}$ & Obrigatório & 15 & 5,70 & 1,46 & \\
$\begin{array}{c}\text { Acordo entre o pacigatório } \\
\text { 0 terapeuta (WSC) }\end{array}$ & 36 & 6,29 & 0,86 & 0,182 \\
& Obrigatório & 15 & 5,48 & 1,43 & 0,141 \\
\hline
\end{tabular}

Análise comparativa entre pacientes em relação à percepção de coerção para tratamento

Num segundo momento, todos os pacientes foram analisados considerando-se a percepção de coerção para realizar psicoterapia. Assim, foram identificados 20 casos em que se sentem obrigados a realizar psicoterapia (percepção de coerção positiva) e 30 casos em que não se sentem obrigados (percepção de coerção negativa) a participar do processo psicoterapêutico. Observase, dessa forma, que há um número maior de pacientes $(N=20)$ com percepção de coerção positiva, comparado ao número total 
de pacientes que, efetivamente, foram encaminhados de forma obrigatória $(\mathrm{GO})$ para psicoterapia $(N=15)$. No grupo obrigatório (GO), somente oito casos (53,3\%) do total de 15 referem ter se sentido obrigados (percepção de coerção positiva) a realizar psicoterapia. Já no grupo não obrigatório (GNO), dentre o total de 36 casos (um paciente não respondeu a questão), 12 casos (34,3\%), referem ter se sentido obrigados a realizar psicoterapia (percepção de coerção positiva). Logo, a percepção de coerção não está associada necessariamente ao fato de ter sido encaminhado de forma obrigatória à psicoterapia, mas a como o paciente percebe a situação de seu encaminhamento. Quanto à percepção de necessidade de psicoterapia, dos 51 pacientes da amostra, apenas um deles afirma acreditar que não necessitava de psicoterapia. Este paciente estava em psicoterapia obrigatória.

\section{Aliança terapêutica}

Os resultados comparativos da Escala de Aliança Psicoterápica da Califórnia (CALPAS-P), conforme ilustra a Tabela 2, indicam que os pacientes que se sentem obrigados (percepção de coerção positiva) diferem daqueles que não se sentem obrigados a realizar psicoterapia (percepção de coerção negativa), em relação à subescala Compreensão e Envolvimento do Terapeuta (TUI). Nesse caso, os pacientes com percepção de coerção positiva apresentaram média inferior $(M=5,61, D P=1,41)$ do que os pacientes com percepção de coerção negativa $(M=6,45, D P=0,65, p=$ $0,024)$ na referida subescala.

Tabela 2

Resultados Comparativos da Escala de Aliança Psicoterápica da Califórnia em Pacientes que se Sentiram e que não se Sentiram Obrigados a Ingressar em Psicoterapia

\begin{tabular}{|c|c|c|c|c|c|}
\hline & Grupo & $\mathrm{N}$ & Média & DP & Significância \\
\hline \multirow{2}{*}{$\begin{array}{l}\text { Escala de Aliança } \\
\text { Psicoterápica da } \\
\text { Califórnia - Total }\end{array}$} & $\begin{array}{l}\text { Sentiu-se } \\
\text { obrigado }\end{array}$ & 19 & 21,33 & 4,49 & \multirow{2}{*}{0,065} \\
\hline & $\begin{array}{l}\text { Não se sentiu } \\
\text { obrigado }\end{array}$ & 30 & 23,38 & 3,08 & \\
\hline \multirow{2}{*}{$\begin{array}{l}\text { Compromisso do } \\
\text { Paciente (PC) }\end{array}$} & $\begin{array}{l}\text { Sentiu-se } \\
\text { obrigado }\end{array}$ & 19 & 5,29 & 1,21 & \multirow{2}{*}{0,237} \\
\hline & $\begin{array}{l}\text { Não se sentiu } \\
\text { obrigado }\end{array}$ & 30 & 5,70 & 1,13 & \\
\hline \multirow{2}{*}{$\begin{array}{l}\text { Capacidade de } \\
\text { trabalho (PWC) }\end{array}$} & $\begin{array}{l}\text { Sentiu-se } \\
\text { obrigado }\end{array}$ & 19 & 4,84 & 1,21 & \multirow{2}{*}{0,491} \\
\hline & $\begin{array}{l}\text { Não se sentiu } \\
\text { obrigado }\end{array}$ & 30 & 5,04 & 0,87 & \\
\hline \multirow{2}{*}{$\begin{array}{l}\text { Compreensão e } \\
\text { envolvimento do } \\
\text { terapeuta (TUI) }\end{array}$} & $\begin{array}{l}\text { Sentiu-se } \\
\text { obrigado }\end{array}$ & 19 & 5,61 & 1,41 & \multirow{2}{*}{$0,024^{*}$} \\
\hline & $\begin{array}{l}\text { Não se sentiu } \\
\text { obrigado }\end{array}$ & 30 & 6,45 & 0,65 & \\
\hline \multirow[t]{2}{*}{$\begin{array}{c}\text { Acordo entre o paciente } \\
\text { e o terapeuta (WSC) }\end{array}$} & $\begin{array}{l}\text { Sentiu-se } \\
\text { obrigado }\end{array}$ & 19 & 5,58 & 1,22 & \multirow[t]{2}{*}{0,082} \\
\hline & $\begin{array}{l}\text { Não se sentiu } \\
\text { obrigado }\end{array}$ & 30 & 6,17 & 1,07 & \\
\hline
\end{tabular}

\section{Escala de sintomas (SCL-90-R)}

A avaliação do instrumento Escala de Sintomas (SCL-90-R) em relação aos pacientes que se sentem obrigados (percepção positiva de coerção) e os pacientes que não se sentem obrigados (percepção negativa), indica não haver diferenças no Índice de Gravidade Geral (IGG) dos sintomas entre os dois grupos, bem como nas escalas de sintomas. O IGG dos sintomas dos pacientes com percepção positiva de coerção é de $M=1,65(D P=0,84$ e dos pacientes com percepção negativa é de $M=1,30(D P=0,65)$.

\section{Mecanismos defensivos (DSQ-40)}

Em adição, comparações foram feitas com os pacientes que se sentem obrigados (percepção positiva de coerção) e pacientes que não se sentem obrigados (percepção negativa de coerção) a realizar psicoterapia, no que tange ao questionário de estilos de defesa. As médias dos pacientes que se sentem obrigados (percepção positiva de coerção) foram: fator maduro (FM) $M=4,68$, $D P=1,31$, fator neurótico (FN) $M=4,35, D P=1,28$ e fator imaturo (FI) $M=3,34, D P=0,91$. Entre os pacientes que não se sentem obrigados (percepção negativa de coerção), as médias foram: fator maduro (FM) $M=4,85, D P=1,52$, fator neurótico (FN) $M$ $=4,47, D P=1,68$ e fator imaturo (FI) $M=3,34, D P=0,91$. Não houve diferença entre os grupos.

\section{Relações objetais}

Em relação ao Object Relations and Reality Testing Inventory (BORRTI - Forma O), não foram encontradas diferenças significativas entre os 20 pacientes que se sentem obrigados (percepção positiva de coerção) e os 30 que não se sentem obrigados (percepção negativa de coerção) a realizar psicoterapia, exceto na dimensão egocentrismo. Nesta dimensão, a frequência de pacientes que se sentem obrigados (percepção positiva de coerção) com escore clínico é de $N=13$ (59\%) e de pacientes que não se sentem obrigados (percepção negativa de coerção) é de $N=9$ (40\%) $\left(X^{2}(1)=5,073\right.$, $p=0,039$ ). Nos 20 pacientes que se sentem obrigados (percepção positiva de coerção) as demais frequências de escores clínicos são respectivamente: 15 (50\%) casos clínicos na dimensão alienação (AL), nove (45\%) vinculação insegura (IN), sete (35\%) incapacidade social (SOC). Por sua vez, a frequência de escore clínico entre os pacientes que não se sentem obrigados nas outras dimensões foi: alienação (AL) 15 (50\%) casos clínicos, 14 (46\%) vinculação insegura (IN), e nove (32\%) incapacidade social (SOC).

\section{Discussão}

O objetivo deste estudo foi identificar e comparar o estabelecimento da AT em pacientes encaminhados à psicoterapia de forma obrigatória, em relação aos pacientes que buscam atendimento psicoterapêutico voluntariamente. Além disso, foram investigados alguns fatores associados ao desenvolvimento da AT, incluindo características: gravidade dos sintomas, mecanismos de defesa e características da percepção de coerção para realizar psicoterapia. Apesar de existirem poucos estudos sobre a AT e a psicoterapia obrigatória (Sotero \& Relvas, 2012), o tema mostra-se bastante relevante, tendo em vista que oferece um conhecimento 
específico para o entendimento do desenvolvimento do vínculo entre paciente e terapeuta na psicoterapia obrigatória.

Inicialmente, é importante destacar que todos os pacientes no presente estudo eram funcionários públicos municipais e estavam em atendimento obrigatório ou não obrigatório na mesma instituição. Esse dado pode ter contribuído para que ambos os grupos se apresentassem homogêneos em relação às características sociodemográficas. Por exemplo, um dado que chama atenção é a predominância maciça do sexo feminino nos dois grupos.

Analisando-se os resultados no grupo obrigatório e não obrigatório, em relação às variáveis investigadas, observou-se que houve similaridade nos dois grupos quanto ao padrão defensivo e à quase totalidade das dimensões das representações objetais, com exceção do egocentrismo que foi mais frequente no grupo obrigatório. Contudo, quanto à sintomatologia, o grupo obrigatório apresentou maior gravidade de sintomas, bem como nas escalas de obssessividade, ansiedade fóbica e hostilidade. Essa diferença está em consonância com o quadro clinico dos pacientes, justificando o afastamento do trabalho e a necessidade de encaminhamento para a psicoterapia obrigatória.

Considerando-se a questão principal deste estudo, ainda que alguns aspectos sintomáticos e intrapsíquicos caracterizassem de maneira mais específica os pacientes obrigatórios e não obrigatórios, as características da AT, média da aliança total, bem como das dimensões avaliadas foram similares nos dois grupos. Logo, independente da gravidade sintomática, padrão defensivo e características das relações objetais, ambos os grupos estabeleceram relações terapêuticas baseadas em um grau de aliança similar. Resultado similar em relação ao funcionamento defensivo do paciente em psicoterapia breve dinâmica, foi verificado por Despland et al. (2001) que constataram que o nível de funcionamento defensivo, isoladamente, não interfere e é menos significante no estabelecimento da aliança inicial.

Entretanto, ao ser considerada a percepção de coerção, o grupo que não se sentiu obrigado a realizar psicoterapia obteve uma pontuação significativamente maior na subescala Compreensão $e$ envolvimento do terapeuta (TUI). Esse resultado indica que a percepção de coerção interfere na forma em que o paciente percebe o terapeuta. No grupo com percepção de coerção positiva o terapeuta pode assumir uma representação de alguém que está julgando e/ou que possui uma menor capacidade de demonstrar compromisso em ajudar, entender o ponto de vista e o sofrimento do paciente. Esse aspecto aponta para um foco importante de trabalho terapêutico, considerando principalmente a necessidade do terapeuta em trabalhar as percepções do paciente que se percebe em obrigação acerca de seu envolvimento no processo.

Outro ponto importante é que a percepção de coerção em relação ao encaminhamento para psicoterapia não se associou necessariamente ao fato de o paciente pertencer ou não ao grupo obrigatório. Por conseguinte, foram identificados no grupo obrigatório $(N=15)$ somente oito casos que afirmaram terem se sentido obrigados a ingressar na psicoterapia. Da mesma forma, no grupo não obrigatório, dentre o total de 35 casos, 12 casos (34,3\%) referiram terem se sentido obrigados a realizar psicoterapia.

Embora o encaminhamento obrigatório esteja fortemen- te associado à percepção de coerção (Iversen et al., 2007), esta informação aponta para um dado inusitado de que pessoas em atendimento não obrigatório podem sentir-se de alguma forma obrigadas a buscar tratamento, mesmo que não tenham sido formalmente encaminhadas para psicoterapia. Estudos sugerem que nem sempre o uso da coerção é evidente no atendimento obrigatório e que não se pode equipará-lo a uma falta de vontade para o tratamento. Além disso, afirmam que a visão do paciente sobre a obrigatoriedade do atendimento pode mudar durante o processo psicoterapêutico (Spence, Goldney, \& Costain, 1988).

Com base em estudos internacionais (Botella \& Corbella, 2011; Horvath \& Luborsky, 1993; Horvath \& Symonds, 1991; Horvath, Del Re, Flückiger, \& Symonds, 2011) que apresentam o estabelecimento de uma AT de qualidade como fator preponderante para o resultado da terapia, pode-se considerar que a não ocorrência de diferenças significativas na AT entre os grupos nesta pesquisa, indica que o estabelecimento da AT na psicoterapia obrigatória pode ser semelhante à buscada de forma voluntária. O status de admissão involuntário parece, pois, não influenciar na aderência e resultado do tratamento (Opjordsmoen et al., 2010).

Outra característica atribuída aos clientes involuntários é que esses pacientes são "resistentes" ao tratamento (Chui \& Ho, 2006). No entanto, como exposto anteriormente, involuntário não é sinônimo de resistência. O fato de os pacientes involuntários terem sido encaminhados por terceiros para o tratamento não pressupõe que estes sejam contra o tratamento e, portanto, resistentes. Segundo Chui e Ho (2006), o termo involuntário refere-se somente aos status de entrada no serviço do cliente, enquanto que 'resistente' refere-se a comportamentos e características que dificultam o processo terapêutico.

\section{Conclusão}

Em suma, considerando os objetivos deste trabalho de identificar o estabelecimento da aliança terapêutica em pacientes encaminhados de forma obrigatória para a psicoterapia em comparação aos pacientes que buscam atendimento psicoterapêutico voluntariamente, observou-se que pacientes encaminhados de forma obrigatória, ou pacientes que procuraram voluntariamente atendimento, não apresentaram diferenças no desenvolvimento da AT. Dessa forma, o status de paciente em psicoterapia obrigatória não impediu o estabelecimento da AT. Ao contrário, a percepção de coerção foi mais influente no sentido de que os pacientes que ao não se perceberem como "obrigados" a permanecer em psicoterapia, tinham uma percepção mais positiva do terapeuta.

Sem dúvida algumas limitações do estudo devem ser apontadas, tal como o tamanho da amostra e a questão da predominância do sexo feminino. Da mesma forma, os casos estudados não se enquadravam em situações envolvendo punições jurídicas ou contravenções, mas se limitavam a casos oriundos de demandas em saúde mental. Entretanto, os resultados indicam que investigações sobre a formação do vínculo terapêutico são necessárias, principalmente nas situações de encaminhamento obrigatório. Portanto, sugere-se a realização de outras pesquisas, considerando 
outros grupos e contextos, de forma a ampliar e investigar o real impacto dos fatores analisados no estabelecimento da AT.

\section{Referências}

Andrews, G., Singh, M., \& Bond, M. (1993). The defense style questionnaire. Journal of Nervous \& Mental Disease, 181(4), 246-256. doi: 10.1097/00005053-199304000-00006

Blaya, C., Kipper, L., Heldt, E., Isolan, L., Ceitlin, L. H., Bond, M., \& Manfro, G. G. (2004). Versão em português do Defense Style Questionnaire (DSQ40) para avaliação dos mecanismos de defesa: Um estudo preliminar. Revista Brasileira de Psiquiatria, 26(4), 255-258. doi: 10.1590/S151644462004000400010

Bordin, E. S. (1979). The generalizability of the psychoanalytic concept of the working alliance. Psychotherapy: Theory, Research and Practice, 16(3), 252260. doi: $10.1037 / \mathrm{h} 0085885$

Botella, L., \& Corbella, S. (2011). Alianza terapêutica evaluada por el paciente y mejora sintomática a lo largo de proceso terapêutico. Boletín de Psicología, 101, 21-33. doi: 10.4067/S0718-48082008000100008

Bruscato W. L., \& Iacoponi, E. (2000). Validade e confiabilidade da versão brasileira de um inventário de avaliação de relações objetais. Revista Brasileira de Psiquiatria, 22(4), 172-7. doi: 10.1590/S151644462000000400006

Byrd, K. R., Patterson, C. L., \& Turchik, J. A. (2010). Working alliance as a mediator of client attachment dimensions and psychotherapy outcome. Psychotherapy, 47(4), 631-636. doi: 10.1037/a0022080 Recuperado de http://www.portalpsicologia.org/servlet/File?idDocumento=3425

Chui, W. H., \& Ho, K. M. (2006). Working with involuntary clients: Perceptions and experiences of outreach social workers in Hong Kong. Journal of Social Work Practice, 20, 205-222. doi: 10.1080/02650530600776947

Despland, J. N., Roten, Y., Despars, J., Stigler, M., \& Perry, J. C. (2001). Contribution of patient defense mechanisms and therapist interventions to the development of early therapeutic alliance in a brief psychodynamic investigation. Journal Psychotherapy Practice Research, 10(3), 155164. Recuperado de http://www.ncbi.nlm.nih.gov/pmc/articles/ PMC3330648/

Derogatis, L. R. (1994). Symptom checklist-90-R (SCL-90-R) administration, scoring, and procedures.Manual. Minneapolis: National Computer Systems.

Frank, D., Perry, J. C., Kean, D., Sigman, M., \& Geagea, K. (2005). Effects of compulsory treatment orders on time to hospital readmission. Psychiatric Services, 56(7), 867-869. doi: 10.1176/appi.ps.56.7.867

Fritz, P. C. (2006). Condenados a terapia: la terapia obligada como estrategia para la modificación de conductas violentas en agresores conyugales. Manuscrito não publicado. Recuperado de: http://www.portalpsicologia. org/servlet/File?idDocumento=3425

Goldman, G., \& Anderson, T. (2007). Quality of object relations and security of attachment as predictors of early therapeutic alliance. Journal of Counseling Psychology, 54(2), 111-117. doi: 10.1037/0022-0167.54.2.111

Gomes, F., Ceitlin, L., Hauck, S., \& Terra, T. (2008). A relação entre os mecanismos de defesa e a qualidade da aliança terapêutica em psicoterapia de orientação analítica. Revista de Psiquiatria do Rio Grande do Sul, 30(2), 109-114. doi: 10.1590/S0101-81082008000300006

Greenson, R. R. (1981). A técnica e a prática em psicanálise. Rio de Janeiro: Imago.

Hersoug, A. G., Amplo, S., Rossberg, O. S., Gabbard, G., Bogwald, K. P., Marble, A.,... Crits-Christoph, P. (2011). Effects of transference work in the context of therapeutic alliance and quality of object relations. Journal of Consulting Clinical Psychology, 79(5), 697-706. doi: 10.1037/a0024863

Horvath, A. O., \& Luborsky, L. (1993). The role of the therapeutic alliance in psychotherapy. Journal of Consulting and Clinical Psychology, 61(4), 561573. doi: 10.1037/0022-006X.47.5.882.

Horvath, A. O., \& Symonds, B. D. (1991). Relation between alliance and outcome in psychotherapy: A meta-analysis. Journal of Counseling Psychology, 38, 139-149. doi: 10.1037/0022-0167.38.2.139.
Horvath, A. O., Del Re, A., Flückiger, C., \& Symonds, D. (2011). Alliance in individual psychotherapy. American Psychological Association, 48(1), 9-16. doi: $10.1037 / \mathrm{a} 0022186$

Iversen, K., Hoyer, G., \& Sexton, H. (2007). Coercion and patient satisfaction on psychiatric acute wards. International Journal of Law and Psychiatry, 30, 504-511. doi: 10.1016/j.ijlp.2007.09.001

Iversen, K., Hoyer, G., Sexton, H., \& Gronli, O. (2002). Perceived coercion among patients admitted to acute wards in Norway. Journal of Cognitive Psychotherapy, 22(1), 68-79. doi: 10.1080/08039480260389352

Kaltiala-Heino, R., Laippala, P., \& Salokangas, R. K. (1997). Impact of coercion on treatment outcome. International Journal of Law and Psychiatry, 20(3), 311-322. doi: 9347394

Kramer, U., Roten, Y., Perry, C., \& Despland, J. (2009). Specificities of defense mechanisms in bipolar affective disorder relations with symptoms and therapeutic alliance. Journal of Nervous and Mental Disease, 197(9), 675681. doi: 10.1097/NMD.0b013e3181b3b224

Marcolino, J. A., \& Iacoponi, E. (2001). Escala de Aliança Psicoterápica da Califórnia na versão do paciente. Revista Brasileira de Psiquiatria, 23(2), 88-95. doi: 10.1590/S1516-44462001000200007

Marziali, E., \& Alexander, L. (1991). The power of therapeutic relationship. American Journal of Orthopychiactry, 61(3), 383-391. doi: 10.1037/ h0079268

Nakhost, A., Perry, C., \& Frank, D. (2012). Assessing the outcome of compulsory treatment orders on management of psychiatric patients at 2 McGill University- Associated Hospitals. Canadian Journal of Psychiatry, 57(6), 359-365. doi: PMID: 22682573

Opjordsmoen, S. F., Melle, I., Haahr, U., Johannessen, J. O., Larsen, T. K.; Rossberg, J. I.,... McGlashan, T. H. (2010). A 2-year follow-up of involuntary admission's influence upon adherence and in firstepisode psychosis. Acta Psychiatrica Scadinavica, 121, 371-376. doi: 10.1111/j.1600-0447.2009.01536.x

Piper, W. E., Ogrodniczuk, J. S., \& Joyce, A. S. (2004). Quality of object relations as a moderator of the relationship between pattern of alliance and outcome in short-term individual psychotherapy. Journal of Personality Assessment, 83(3), 345-356. doi: 10.1207/s15327752jpa8303_15

Reis, T., \& Ramos M. (2010). Trabalho psicológico compulsório com homens envolvidos em violência doméstica. Psicologia IESB, 2(1), 70-80.

Ribeiro, M. A., Costa, L. F., Penso, M. A., Almeida, T. C., \& Nogueira, H. F. (2010). O grupo multifamiliar em parceria com a ação psicossocial forense. Interação em Psicologia, 14(1), 73-82. doi: 10.1590/S010356652011000100012

Sotero, L., \& Relvas, A. P. (2012). A intervenção com clientes involuntários: Complexidade e dilemas. Psicologia \& Sociedade, 24(1), 187-196. doi: 10.1590/S0102- 71822012000100021

Spence, N. D., Goldney, R. D., \& Costain, W. F. (1988). Attitudes toward psychiatric hospitalization: A comparison of involuntary and voluntary patients. Australian Clinical Review, 8, 108-116. doi: PMID: 3228391

Vans, H. L., Hendriksen, M., Schoevers, R. A., Peen, J., Abraham, R. A., \& Dekker, J., (2008). Predictive value of object relations for therapeutic alliance and outcome in psychotherapy for depression: An exploratory study. Journal of Nervous \& Mental Disease, 196(9), 655-662. doi: 10.1097/ NMD.0b013e318183f8c2

Viadel, M., Nicolás, C., Prieto, J. F., Calatayud, V., \& Beneyeto, M. (2010). Evaluación de la eficácia del tratamiento ambulatório involuntário para reducir la atención hospitalaria. Revista de Psiquiatria y Salud Mental, 3(2), 50-54. doi: 10.1016/j.rpsm.2010.03.001

Yoshida, E. (2001). Psicoterapia breve dinâmica: Critérios de indicação. Psicologia-Teoria e Prática, 3(1), 43-51. Recuperado de http:// editorarevistas.mackenzie.br/index.php/ptp/article/view/1099 
Natacha Hennemann de Oliveira Becker, Mestre em Psicologia Clínica pela Universidade do Vale do Rio dos Sinos, UNISINOS, é Psicóloga da Clínica Cruz e Hennemann Médicos e Associados. Endereço para correspondência: Rua Independência, $n^{\circ} 181$, sala 1204, Centro - São Leopoldo, RS. CEP 93.010-050. Telefone (51) 92597188. E-mail: natacha.h@hotmail.com

Sílvia Pereira da Cruz Benetti, Doutora em Psicologia Syracuse University, USA, Pós-Doutorado na Zurich University of Applied Sciences ( Zhaw), é Professora Adjunto do Programa de Pós-Graduação em Psicologia da Universidade do Vale do Rio dos Sinos, UNISINOS. E-mail: spcbenetti@gmail.com 